\title{
Access to Care and Worsening Eating Disorder Symptomatology during the COVID-19 Pandemic
}

Rebecca Spigel ( $\nabla$ rebecca.spigel@childrens.harvard.edu )

Boston Childrens Hospital: Boston Children's Hospital https://orcid.org/0000-0002-8116-9144

Jessica Lin

Boston Childrens Hospital: Boston Children's Hospital

Carly E Milliren

Boston Childrens Hospital: Boston Children's Hospital

Melissa Freizinger

Boston Childrens Hospital: Boston Children's Hospital

Julia A Vitagliano

Boston Childrens Hospital: Boston Children's Hospital

Elizabeth R Woods

Boston Childrens Hospital: Boston Children's Hospital

\section{Sara F Forman}

Boston Childrens Hospital: Boston Children's Hospital

Tracy K Richmond

Boston Childrens Hospital: Boston Children's Hospital

\section{Research Article}

Keywords: COVID-19, Eating disorders, adolescents, access, telemedicine

Posted Date: March 16th, 2021

DOl: https://doi.org/10.21203/rs.3.rs-293019/v1

License: (a) (1) This work is licensed under a Creative Commons Attribution 4.0 International License.

Read Full License 


\section{Abstract}

\section{Background}

Shelter-in-place orders and social distancing guidelines, in response to the COVID-19 pandemic, have limited traditional face-to-face interactions and led to many clinical providers transitioning to the use of videoconferencing platforms. The present study aims to assess how the COVID-19 pandemic has impacted adolescents'/young adults' (AYA) eating disorder (ED)-related care, and how access to, changes in, perceived disruptions to, and quality of care are associated with ED thoughts and behaviors.

\section{Methods}

AYA enrolled in the RECOVERY study, a pre-existing web-based longitudinal study, completed a COVID-19specific survey $(n=89)$. We examined bivariate associations of four markers of care: access to and changes in care as well as perceived disruption to and quality of care. Using multiple logistic regression, we examined the associations of our pandemic-related markers of care with changes in ED thoughts and behaviors. We excluded those not engaged in treatment pre-pandemic $(n=16)$.

Results

In the remaining 73 participants, reported access to care was high, with $92 \%$ of respondents continuing to see at least one ED provider during the pandemic; however, $47 \%$ stopped some treatment during the pandemic. Nearly one-third (32\%) perceived a disruption in treatment. Quality of care remained high with $67 \%$ reporting care to be better than or as good as pre-pandemic. Respondents acknowledged heightened symptomatology: $81 \%$ reported increased ED thoughts and $81 \%$ reported increased ED behaviors due to COVID-19-related factors. However, none of the markers of care described were significantly associated with ED thoughts or behaviors in regression analyses adjusting for demographic variables and baseline characteristics, except our quality of care measure which was approaching significance $(p=0.07)$.

\section{Conclusions}

Our findings show the majority of AYA who had care prior to the pandemic are still receiving some element of their multi-disciplinary ED treatment and perceive their care as high quality. Although none of the markers of care described were statistically associated with increased ED thoughts and behaviors, our results may indicate that continued access to care may be protective against increased ED behaviors and patients who perceive their quality of care as worse than usual might be more likely to have intrusive ED thoughts.

\section{Plain English Summary}

The COVID-19 pandemic has had a negative impact on our global community's mental health, in particular those struggling with psychiatric illnesses, such as eating disorders (ED). Stay-at-home orders 
have transitioned to using videoconferencing platforms. Clinicians who care for patients with EDs worry that these sudden changes in accessing treatment, on top of mental health challenges associated with the pandemic, may contribute to worsening ED symptoms. In this study, we asked adolescent and young adult patients with EDs about their symptoms, access to treatment, changes in care, disruptions in treatment and quality of care since the pandemic started. Our results demonstrate that patients with established care teams have maintained treatment and perceive their care as high quality, though the majority are experiencing worsening ED thoughts and behaviors. Patients who perceive their quality of care as worse than usual might be more likely to have intrusive ED thoughts. Continued access to care could also be protective against increased ED behaviors. Ultimately, our study highlights the need for continued support of patients during this challenging time.

\section{Background}

Since the beginning of 2020, the COVID-19 pandemic has had a profound impact on youth around the world. Though these effects have been universal, individuals with pre-existing psychiatric illnesses are especially vulnerable to the consequences of COVID-19. ${ }^{1}$ Individuals with eating disorders (ED) are at particular risk, as stress and anxiety may lead to worsening eating disordered cognitions, which may further lead to negative behaviors and detrimental physical effects. ${ }^{2}$ Not surprisingly, individuals with EDs have reported increased social isolation, rumination about eating, feelings of anxiety and depression, and decreased feelings of control and social support during the COVID-19 pandemic. ${ }^{3-5}$ Clinicians working with patients with EDs have also voiced concern that the changes caused by COVID-19 may increase ED symptoms, decrease protective factors, and exacerbate barriers to care. ${ }^{6}$

The standard of care for ED treatment involves a multi-disciplinary team to address the medical, psychological, and nutritional components of this serious, life-threatening psychiatric illness. ${ }^{7}$ Shelter-inplace orders and social distancing guidelines have limited face-to-face interactions, leading to residential and day program closures and limiting in-person outpatient care from all disciplines. Thus, for most patients there have been reductions in in-person contact with clinical teams. ${ }^{8}$ Recognizing the need to limit in-person clinical care, governing bodies responded by changing national and state payment policies and lifting technology restrictions, encouraging clinicians to maintain treatment continuity. ${ }^{9}$ In response, providers across disciplines have rapidly transitioned their practices to videoconferencing platforms, commonly referred to as telehealth. ${ }^{10,11}$ ED clinicians have published recommendations on how to adapt multi-disciplinary ED care to telehealth, ${ }^{12-14}$ however, little is known about how patients perceive this change in their care as a result of the COVID-19 pandemic.

Experts worry that these sudden changes and limited access to in-person care, coupled with increasing mental health challenges associated with the pandemic, may have serious implications for patients with EDs. ${ }^{3}$ The absence of in-person care creates specific challenges for ED treatment, namely, monitoring for changes in weight and vital signs, essential markers of illness. ${ }^{14,15}$ Additionally, some believe that the 
telehealth. ${ }^{16}$ Though many raise concerns that reduction in in-person access to clinical providers may contribute to the worsening of ED symptoms, little research currently exists. ${ }^{8,12,13,17}$ Thus, we set out to do the following: 1) examine four markers of care during the time of the COVID-19 pandemic: reported access to (telehealth and/or in-person), changes to, perceived disruption of, and reported quality of EDrelated care; and 2) examine whether these four markers of care are associated with increased ED thoughts and behaviors. We hypothesized that decreased access to, changes in, perceived lower quality of, as well as perceived disruption to care would be associated with increased ED thoughts and behaviors.

\section{Methods}

\section{Study Sample}

We used data from the Registry of Eating Disorders and their Co-Morbidities Over Time in Youth (RECOVERY), a longitudinal registry of adolescent/young adult patients ages 10-27 (average age, 17.1 years at enrollment) seeking ED treatment. RECOVERY study participants were previously recruited from the outpatient ED program at Boston Children's Hospital from June 2017 to February 2020.

Participants provided written consent and assent prior to enrollment.

Using web-based surveys sent via Research Electronic Data Capture (REDCap a HIPAA compliant database) patients, their parents, and their clinicians answered questions regarding ED behaviors and treatment every three months for the first year and every six months thereafter. Participants received remuneration for each completed survey.

In July 2020, in response to the COVID-19 pandemic, we invited all patient participants to complete an added survey with the aim of understanding the impact of the COVID-19 pandemic on ED treatment, behaviors, and general well-being.

The COVID-19 survey was separate from the regularly scheduled RECOVERY surveys, and participants were informed that additional remuneration was not available.

The RECOVERY study and the additional COVID-19 survey were approved by the Boston Children's Hospital Institutional Review Board.

\section{Survey Measures}

Sample demographic characteristics were obtained from the participants' baseline RECOVERY surveys. Information regarding treatment access, perceived disruption of, reported changes to, and perceived quality of care, as well as the effects of the COVID-19 pandemic on ED thoughts and behaviors, were obtained from the COVID-19 Survey. This survey was adapted from one developed at the University of North Carolina Center of Excellence for Eating Disorders. ${ }^{3}$

\section{Primary predictor variables}


Access to Care. Participants were asked about their access to multi-disciplinary ED care since the pandemic via the question, "I have been able to access my providers...". Participants could then check all that applied: "via telehealth," "in person," "neither (I have not been able to access my providers at all)." Responses were then dichotomized to indicate any access to care (via telehealth or in-person) vs. no access.

\section{Changes to Care}

Participants were asked about changes in different elements of their care in a series of two questions. First, respondents were asked to report what care they were receiving prior to the pandemic (i.e., "Before the pandemic, I was involved in..."). Participants could then check all that applied from weight checks, nutrition, or therapy appointments. Participants were then asked to report on the care they have received since the pandemic (i.e., "Since the pandemic, I have been involved in..."), and were asked to check all that applied from the same list of potential appointments. We then compared the pre- and post-pandemic reported care and created a variable indicating any stopped care (if any of the three -weight checks, therapy, or nutrition-changed from pre- to post-pandemic) v. no change to care.

Quality of Care: Patients were asked to rate the overall quality of their care they received during the first few months of the pandemic, compared to care received prior to the pandemic, using a 4-point Likert scale: "better than usual", "as good as usual", "somewhat worse than usual," and "worse than usual".

\section{Perceived Treatment Disruption}

Patients were asked to report whether their overall ED treatment had been disrupted as a result of the COVID-19 pandemic with response options of yes, no, not applicable.

\section{Primary outcomes}

\section{Intrusive ED thoughts and behaviors}

Participants were asked, "how has the COVID-19 pandemic affected intrusive eating disorder thoughts" using a 5-point Likert scale, ranging from "increased significantly" to "decreased significantly." Responses were dichotomized to indicate no increase vs. any increase in ED thoughts. Respondents also reported in three separate questions whether they had engaged in more restrictive behaviors, compensatory behaviors, or binging behaviors in the past three months "because of COVID-19 related factors." Answers to these three questions were on a 4-point Likert scale ranging from "not at all" to "daily or more." Responses from the three questions were combined and then dichotomized to indicate frequently or daily vs. never or rarely for any of the three ED behaviors.

\section{Additional variables}

Age

Loading [MathJax]/jax/output/CommonHTML/jax.js 
Age at time of COVID-19 survey completion was calculated from the date of survey completion and the date of birth obtained from the RECOVERY study baseline survey.

\section{Sex}

Self-reported sex assigned at birth (female, male or another sex) was obtained at the time of RECOVERY study baseline survey completion.

Race/Ethnicity: Patients were asked to select all that applied from the following options on the RECOVERY baseline survey: Hispanic/Non-Hispanic, American Indian or Alaska Native, Asian, Black or African American, Middle Eastern/North African, Native Hawaiian or other Pacific Islander, White/Caucasian or another race. We constructed a mutually exclusive race/ethnicity variable consisting of non-Hispanic white, non-Hispanic Black or African-American, Asian, Multiracial, Other race, and Hispanic.

ED Diagnosis: Patients were asked to select all that applied from the following options on the RECOVERY baseline survey: anorexia nervosa (AN), atypical anorexia nervosa (AAN), Avoidant Restrictive Food Intake Disorder (ARFID), bulimia nervosa (BN), binge-eating disorder (BED), purging disorder, other eating issue(s)/disorder(s), and I don't know/Unsure. AN and AAN were collapsed to create an indicator for restrictive diagnoses vs. other ED diagnoses.

\section{Length of treatment}

This variable was calculated from the date of patient's first ED clinic appointment to the date of COVID-19 survey completion.

\section{Statistical Analysis}

We examined frequencies (percent) for categorical variables and means (standard deviation) for continuous variables. We compared responders to the COVID-19 survey to non-responders from the RECOVERY cohort on demographic factors (age, race/ethnicity and sex assigned at birth) and ED diagnosis using $t$-tests for continuous variables and $\chi^{2}$ tests for categorical variables. Multivariable logistic regression was used to examine the associations of access to, changes in, quality of, and perceived disruption to ED care during the COVID-19 pandemic with changes in ED thoughts and behaviors. Regression analyses were adjusted for age, sex at birth, race/ethnicity, ED diagnosis and length in treatment. All analyses were conducted using SAS (v9.4; Cary, NC).

\section{Results}

\section{Sample Characteristics}

Of the 161 participants enrolled in the RECOVERY study, eighty-nine (55\%) participants responded to the did not differ from non-respondents on age at enrollment, 
race/ethnicity, sex assigned at birth. Participants with restrictive ED diagnoses (AN and AAN) were more likely to respond to the survey $(p=0.03)$ compared to those with non-restrictive diagnoses (BED, BN, ARFID, purging disorder, other eating issue(s)/disorder(s), or I don't know/Unsure).

Of the eighty-nine respondents, sixteen (18\%) were excluded from further analysis because they were not engaged in treatment prior to the pandemic. Of these remaining 73 participants, the mean age was $19.1 \pm$ 3.0 years (Table 1$)$. The majority were female $(93 \%)$, White/non-Hispanic $(79 \%)$ and living with a restrictive ED diagnosis (85\%). Approximately half (53\%) had been in treatment for two or more years.

Table 1

Demographic characteristics, eating disorder diagnosis and report of ED symptomatology of RECOVERY participants who completed the COVID-19 survey $(\mathrm{N}=73)$.

\begin{tabular}{|ll|}
\hline & $\begin{array}{l}\text { Overall } \\
(\mathbf{N}=73)\end{array}$ \\
\hline Age at survey completion (years), mean (SD) & $19.1(3.0)$ \\
\hline Age over 18 at survey completion & $48(66 \%)$ \\
\hline Female at birth & $68(93 \%)$ \\
\hline Race/Ethnicity & \\
\hline White, non-Hispanic & $58(79 \%)$ \\
\hline Asian & $5(7 \%)$ \\
\hline Hispanic & $4(6 \%)$ \\
\hline Black & $1(1 \%)$ \\
\hline Multiracial & $4(6 \%)$ \\
\hline Other race & $1(1 \%)$ \\
\hline Restrictive Eating Disorder Diagnosis & $62(85 \%)$ \\
\hline Length of ED Treatment & \\
\hline$<1$ year & $8(11 \%)$ \\
\hline $1-2$ years & $26(36 \%)$ \\
\hline 2 years or more & $39(53 \%)$ \\
\hline Intrusive ED thoughts as a result of CoVID-19 & $59(81 \%)$ \\
\hline ED Behaviors as a result of COVID-19 & $59(81 \%)$ \\
\hline
\end{tabular}

\section{Access to, Changes in, Quality of, and Perceived Disruptions}


Access to care remained high, with $92 \%$ of respondents reporting continued access to at least one provider via telehealth or in person (Table 2). Of those continuing in care, $88 \%$ used telehealth to see at least one provider. However, nearly half (47\%) of the 73 participants who were actively engaged in ED care prior to the pandemic reported stopping at least one aspect of their ED treatment: sixteen (22\%) stopped mental health counseling/therapy, seven (10\%) stopped nutrition visits, and twenty-three (32\%) stopped weight checks with their medical provider. Approximately one-third (32\%) perceived a disruption in treatment. Of those with telehealth access $(n=64), 9 \%$ found care to be better than usual, $59 \%$ as good as usual, while $30 \%$ said somewhat worse than usual, and $2 \%$ much worse. There was no association between access to care via telehealth and perceived disruption of care $(p=0.99)$ or quality of care $(p=$ $0.36)$, however respondents who perceived a disruption to their treatment were more likely to report lower quality of care $(p=0.004)$. 
Table 2

Markers of care and unadjusted association with ED thoughts and behaviors during the COVID-19 pandemic $(\mathrm{N}=73)$

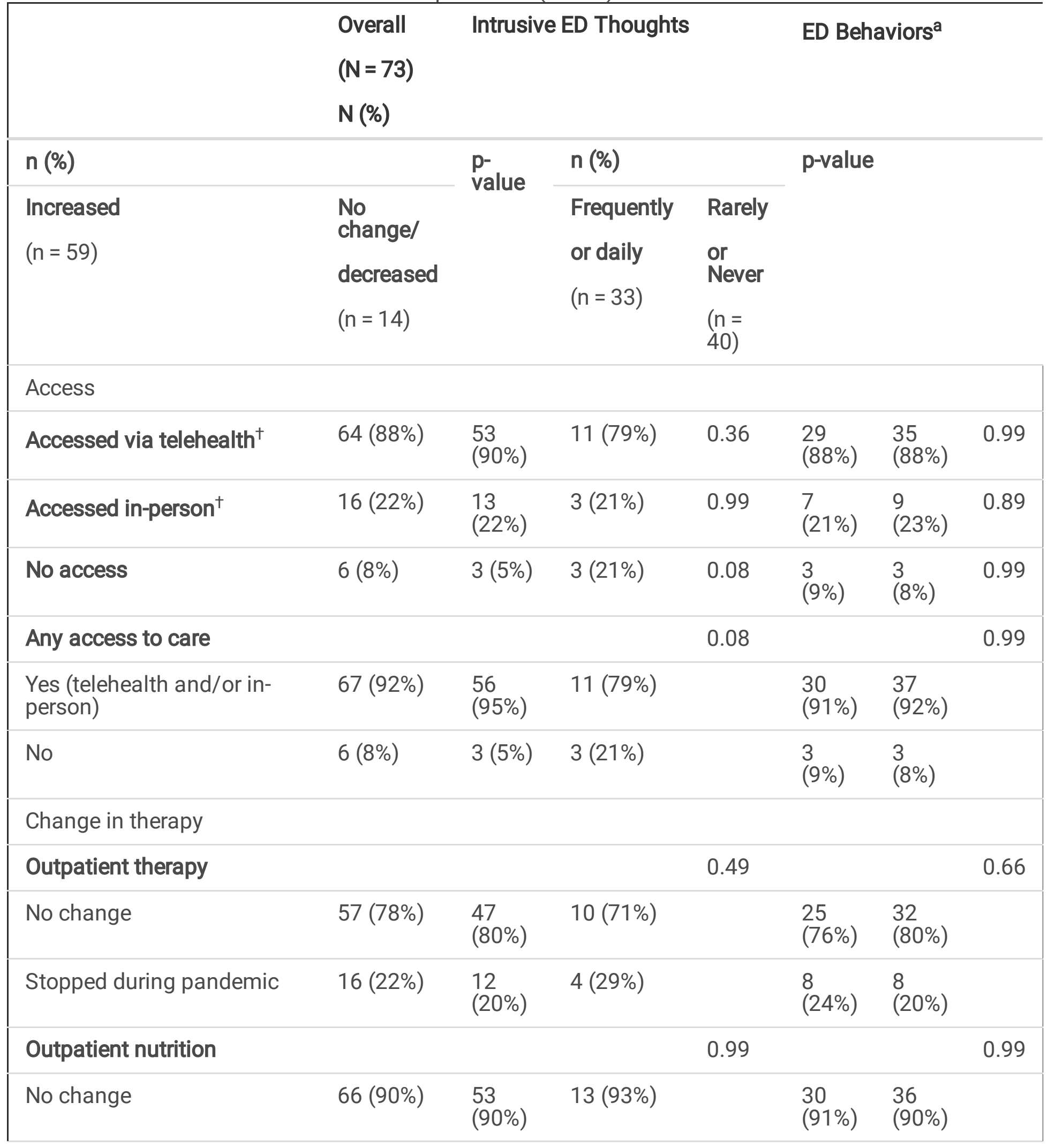

t not mutually exclusive $-\mathrm{n}=13$ reported access both via telehealth and in-person.

a Composite measure for enqaqing in restrictive or compensatory behaviors or binging on food in the Loading [MathJax]/jax/output/CommonHTML/jax.js 


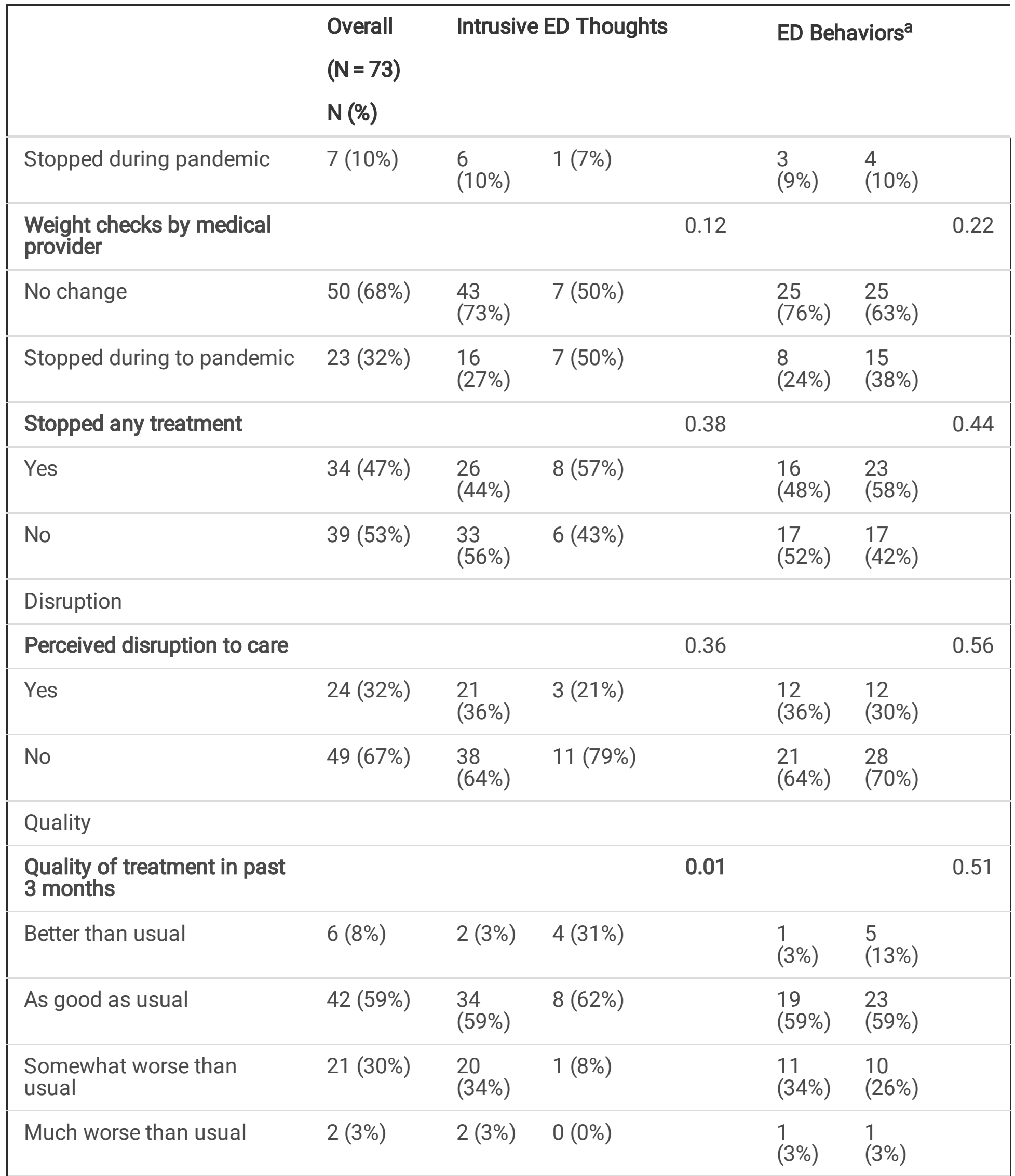

† not mutually exclusive $-n=13$ reported access both via telehealth and in-person.

a Composite measure for engaging in restrictive or compensatory behaviors or binging on food in the past 3 months. 


\section{ED Thoughts and Behaviors}

Many respondents (81\%) endorsed increased intrusive ED thoughts as a result of the COVID-19 pandemic. The same percentage of participants (though not necessarily the same patients) reported an increase in ED behaviors: engaging in restrictive or compensatory behaviors or binging on food at least once in the past three months because of COVID-19-related factors. Thirty-three (45\%) participants reported engaging in the restrictive/compensatory/or binging behaviors frequently or daily.

The association of access to, changes in, perceived quality of and perceived disruption to care with ED Thoughts and Behaviors

Unadjusted and adjusted associations between access to, changes in, perceived quality of and perceived disruption to care, with ED thoughts and behaviors are presented in Table 3. After adjusting for age, sex assigned at birth, race/ethnicity, restrictive ED diagnosis and length of treatment, those who perceived treatment disruption had higher odds of intrusive ED thoughts (adjusted odds ratio $=2.63 ; 95 \% \mathrm{Cl}$ : 0.5612.3) and increased $\mathrm{ED}$ behaviors ( $\mathrm{aOR}=1.98 ; 95 \% \mathrm{Cl}$ : 0.63-6.19) though these associations were not statistically significant. Those who still had access to ED care had higher odds of intrusive ED thoughts $(\mathrm{aOR}=5.32 ; 95 \% \mathrm{Cl}: 0.72-39.6)$ but lower odds of increased ED behaviors (aOR $=0.67 ; 95 \% \mathrm{Cl}: 0.11-$ 4.20), though these associations were not statistically significant. Those who stopped some treatment had lower odds of intrusive ED thoughts ( $\mathrm{aOR}=0.76 ; 95 \% \mathrm{Cl}$ : $0.20-2.88)$, but higher odds of increased ED behaviors $(\mathrm{aOR}=2.05 ; 95 \% \mathrm{Cl}: 0.70-6.05)$, though these associations were not statistically significant. Those who perceived quality of care as worse than usual were more likely to have intrusive ED thoughts $(\mathrm{aOR}=8.00 ; 95 \% \mathrm{Cl}: 0.85-75.7)$, though these associations were approaching significance $(p=0.07)$.

Table 3. Unadjusted and adjusted odds of ED thoughts or behaviors associated with markers of care $(\mathrm{N}=73)$.

Model adjusts for age, sex at birth, race/ethnicity, restrictive diagnosis and length of treatment. 


\begin{tabular}{|c|c|c|c|c|c|c|c|c|}
\hline \multirow[t]{4}{*}{ Predictor $^{\mathrm{a}}$} & \multicolumn{8}{|c|}{ Outcome } \\
\hline & \multicolumn{5}{|c|}{ Intrusive ED Thoughts ${ }^{b}$} & \multicolumn{3}{|c|}{ ED Behaviors ${ }^{c}$} \\
\hline & \multicolumn{2}{|c|}{ Unadjusted } & \multicolumn{2}{|c|}{ Adjusted $^{d}$} & \multicolumn{2}{|c|}{ Unadjusted } & \multicolumn{2}{|c|}{ Adjusted $^{d}$} \\
\hline & $\begin{array}{l}\text { OR } \\
(95 \% \\
\mathrm{Cl})\end{array}$ & $\begin{array}{l}\mathrm{p}- \\
\text { value }\end{array}$ & $\begin{array}{l}\text { OR } \\
(95 \% \\
\mathrm{Cl})\end{array}$ & $\begin{array}{l}\mathrm{p}- \\
\text { value }\end{array}$ & $\begin{array}{l}\text { OR } \\
(95 \% \\
\mathrm{Cl})\end{array}$ & $\mathrm{p}$-value & $\begin{array}{l}\text { OR } \\
(95 \% \\
\mathrm{Cl})\end{array}$ & $\begin{array}{l}\mathrm{p}- \\
\text { value }\end{array}$ \\
\hline $\begin{array}{l}\text { Perceived } \\
\text { treatment } \\
\text { disruption }\end{array}$ & & 0.32 & & 0.22 & & 0.57 & & 0.24 \\
\hline Yes & $\begin{array}{l}2.03 \\
(0.51 \\
8.08)\end{array}$ & & $\begin{array}{l}2.63 \\
(0.56 \\
12.3)\end{array}$ & & $\begin{array}{l}1.33 \\
(0.50 \\
3.55)\end{array}$ & & $\begin{array}{l}1.98 \\
(0.63 \\
6.19)\end{array}$ & \\
\hline No & $\begin{array}{l}1.0 \\
\text { (Ref.) }\end{array}$ & & $\begin{array}{l}1.0 \\
\text { (Ref.) }\end{array}$ & & $\begin{array}{l}1.0 \\
\text { (Ref.) }\end{array}$ & & $\begin{array}{l}1.0 \\
\text { (Ref.) }\end{array}$ & \\
\hline Any access to care & & 0.06 & & 0.11 & & 0.81 & & 0.67 \\
\hline Yes & $\begin{array}{l}5.09 \\
(0.91 \\
28.6)\end{array}$ & & $\begin{array}{l}5.32 \\
(0.72 \\
39.6)\end{array}$ & & $\begin{array}{l}0.81 \\
(0.15 \\
4.31)\end{array}$ & & $\begin{array}{l}0.67 \\
(0.11 \\
4.20)\end{array}$ & \\
\hline No & $\begin{array}{l}1.0 \\
\text { (Ref.) }\end{array}$ & & $\begin{array}{l}1.0 \\
\text { (Ref.) }\end{array}$ & & $\begin{array}{l}1.0 \\
\text { (Ref.) }\end{array}$ & & $\begin{array}{l}1.0 \\
\text { (Ref.) }\end{array}$ & \\
\hline $\begin{array}{l}\text { Stopped any } \\
\text { treatment }\end{array}$ & & 0.38 & & 0.68 & & 0.44 & & 0.19 \\
\hline Yes & $\begin{array}{l}0.59 \\
(0.18 \\
1.92)\end{array}$ & & $\begin{array}{l}0.76 \\
(0.20 \\
2.88)\end{array}$ & & $\begin{array}{l}1.44 \\
(0.57 \\
3.63)\end{array}$ & & $\begin{array}{l}2.05 \\
(0.70 \\
6.05)\end{array}$ & \\
\hline No & $\begin{array}{l}1.0 \\
\text { (Ref.) }\end{array}$ & & $\begin{array}{l}1.0 \\
\text { (Ref.) }\end{array}$ & & $\begin{array}{l}1.0 \\
\text { (Ref.) }\end{array}$ & & $\begin{array}{l}1.0 \\
\text { (Ref.) }\end{array}$ & \\
\hline $\begin{array}{l}\text { Quality of } \\
\text { treatment }\end{array}$ & & 0.06 & & 0.07 & & 0.41 & & 0.37 \\
\hline $\begin{array}{l}\text { As good or } \\
\text { better than usual }\end{array}$ & $\begin{array}{l}1.0 \\
\text { (Ref.) }\end{array}$ & & $\begin{array}{l}1.0 \\
\text { (Ref.) }\end{array}$ & & $\begin{array}{l}1.0 \\
\text { (Ref.) }\end{array}$ & & $\begin{array}{l}1.0 \\
\text { (Ref.) }\end{array}$ & \\
\hline $\begin{array}{l}\text { Somewhat or } \\
\text { much worse than } \\
\text { usual }\end{array}$ & $\begin{array}{l}7.33 \\
(0.89 \\
60.3)\end{array}$ & & $\begin{array}{l}8.00 \\
(0.85 \\
75.7)\end{array}$ & & $\begin{array}{l}1.53 \\
(0.56 \\
4.15)\end{array}$ & & $\begin{array}{l}1.65 \\
(0.55 \\
4.88)\end{array}$ & \\
\hline
\end{tabular}

Abbreviations: OR, odds ratio; $\mathrm{Cl}$, confidence interval

a Separate models predicting outcome from each predictor.

$\mathrm{b}$ Intrusive ED thouahts increased vs. no change/decreased. Loading [MathJax]/jax/output/CommonHTML/jax.js 
c Composite measure for engaging in restrictive or compensatory behaviors or binging on food frequently or daily vs. never or rarely in the past 3 months.

d Models adjusting for age, sex at birth, race/ethnicity, restrictive diagnosis and length of treatment

\section{Discussion}

This study examined the association between markers of care and ED thoughts and behaviors in youth, five months after Massachusetts enacted stay-at-home orders due to the COVID-19 pandemic. Our participants reported marked increases in both intrusive ED thoughts and behaviors, which they attributed to the COVID-19 pandemic. Reassuringly, the majority of our cohort was able to maintain access to at least one member of their care team via telehealth and some were able to continue to see their providers in person. However, half had to stop some aspect of their treatment because of the pandemic. Despite these changes, patient-reported quality of care did not suffer, and most patients did not perceive a disruption to their care. Those who perceived a treatment disruption to care were more likely to rate their care lower than those who did not perceive a disruption. None of the markers of care described was statistically associated with increased ED thoughts and behaviors, though our results may indicate that patients who perceive their quality of care as worse than usual are more likely to have intrusive ED thoughts.

Like many institutions across the globe, ${ }^{11,18-20}$ our clinicians transitioned their practices to videoconferencing platforms shortly after stay-at-home advisories were issued in March 2020. ${ }^{23}$ Despite providers' concerns about patients' perceptions of adapting evidence-based ED treatment to telehealth, ${ }^{12-}$ 14,21 the majority of our patients who accessed their care via telehealth felt their quality of care was as good as, or better, than usual. Previous research has shown telehealth to be effective for use in psychotherapy, ${ }^{22,23}$ as well as effective in adolescent/young adult populations, ${ }^{24-26}$ however little research exists examining the use of this technology for multi-disciplinary ED treatment. ${ }^{27,28}$ Although more research is necessary to determine efficacy, ${ }^{4,29}$ our findings suggest providing ED care via telehealth is well accepted and provides good quality of care for ED patients. Moreover, the expansion and continued use of telehealth for multi-disciplinary ED treatment could mitigate barriers to care and increase access for patients who are less connected or away for college or employment, even after the restrictions of the pandemic are lifted. ${ }^{30}$

Similar to other studies, our patients with EDs reported increases in ED thoughts and behaviors related to the COVID-19 pandemic. ${ }^{3,5,31,32}$ One study attributed an increase in ED thoughts during the COVID-19 pandemic to the lack of in-person care and absence of distractions. ${ }^{33}$ Our findings suggest that continued access to care may be associated with more intrusive ED thoughts, but also could have a protective effect on actual ED behaviors. Although our multivariate analyses were not statistically significant, the trends noted could have clinical implications. Treatment may serve as a protective factor, preventing an increase in ED behaviors. This finding is comparable to another study by Schlegl and colleagues, who found an 
increase in ED cognitions, but not an increase in behaviors during the initial months of the COVID-19 pandemic. ${ }^{5}$ It is critical to understand how to support our patients so that they may remain resilient against their ED behaviors, particularly during these times of stress and isolation, which are known risk factors for patients with EDs. ${ }^{33-37}$ Based on our results, future research should continue to explore how access to care may mitigate ED symptomatology.

One limitation of the present study was the total number of respondents, which decreased the power to address our primary question.

Post-hoc power calculations coupled with wide confidence intervals suggest that we were underpowered to detect statistically significant associations between our markers of care and ED symptomatology, due to the high prevalence of the symptomatology and moderately small sample size.

Given the effect size we observed for the association between access to care and intrusive ED thoughts, we had $66 \%$ power to detect a statistically significant difference. We observed significant associations between our quality of care measure and intrusive ED thoughts in bivariate analysis, though this effect was attenuated after adjustment for other factors. It is likely our findings may be a conservative estimate of the true association: those who perceive quality of care as worse than usual are more likely to have intrusive ED thoughts. An additional limitation is the response rate; only half of participants in the RECOVERY study responded to the COVID-19 survey. There is a possibility that those who did not respond were affected by COVID-19 differently than those who did respond, which could affect the results of this study. Notably, this survey was sent out of sequence and remuneration was not available for completion of this survey, which may explain some loss of respondents. Overall, there were no differences between survey respondents and non-respondents in age, race/ethnicity, sex, but those with restrictive EDs were more likely to respond to the survey.

An additional limitation is the generalizability of our findings. The majority of our study participants identified as white and were diagnosed with restrictive EDs. Future research should examine the differences in markers of care by ED diagnosis and demographic groups, particularly with respect to race/ethnicity and socioeconomic status. Additionally, our participants may be more representative of individuals who were already engaged in care and connected to their health care team, as most participants were in treatment and the majority had been in treatment for more than two years. All the participants in this cohort had providers with ED expertise at some point, making it an easier transition to COVID-19 modified treatment. Published literature has reported increased ED incidence as a result of the pandemic, ${ }^{38}$ which is consistent with our clinical experience during this time. Anecdotally, we have noted that it is especially difficult for these new patients to find providers. Thus, our findings may not be generalizable to patients with new onset EDs and therefore it is critical that we examine the effect of COVID-19 on the incidence of EDs and access to care for new patients.

\section{Conclusions}


The present study demonstrates the profound impact COVID-19 has had on patients' ED symptomatology and access to treatment. Our findings suggest that patients with established care teams prior to the pandemic were able to maintain some aspect of their care via telehealth and perceived it to be high quality. Our patients with EDs reported marked increases in intrusive ED thoughts and behaviors because of the pandemic. Disruption in, decreased quality of, changes to and decreased access to care were all associated with increased ED symptomatology, though these associations were not significant likely due to lack of statistical power. More work is needed to better understand the relationship between care changes and ED symptomatology in larger populations. In the meantime, providers should continue to support patients via telehealth, and in person when possible, who are at risk of worsening EDs during this difficult time.

\section{Abbreviations}

ED - Eating disorder

AN - Anorexia nervosa

AYA - Adolescents'/young adults'

RECOVERY - Registry of Eating Disorders and their Co-Morbidities Over Time in Youth

AAN - Atypical anorexia nervosa

ARFID - Avoidant Restrictive Food Intake Disorder

BN - Bulimia nervosa

BED - Binge-eating disorder

\section{Declarations}

\section{Ethics approval and consent to participate}

The Boston Children's Hospital Institutional Review Board reviewed and approved this study.

\section{Consent for publication}

$N / A$

\section{Availability of data and materials}

The datasets generated and/or analyzed during the current study are not publicly available due protection of patient privacy but are available de-identified from the corresponding author on reasonable request and IRB approval. 


\section{Competing interests}

The authors have no conflicts of interest to disclose.

\section{Funding}

This work was supported in part by the Appleby Family Charitable Fund [95259];

Children's Hospital - Boston PPSQ [96204]; The McCarthy Family Fund; and by the Health

Resources and Services Administration (HRSA) of the U.S. Department of Health and Human

Services (HHS) as part of a MCHB T71MC00009 LEAH training grant. The contents are those of the authors and do not necessarily represent the official views of, nor an endorsement by, HRSA, HHS, or the U.S. Government. For more information, please visit HRSA.gov.

\section{Authors' contributions}

RS designed the study, analyzed and interpreted the data, and was a major contributor in writing the manuscript. JL and CM assisted with study design, data analysis, data interpretation, and were major contributors to the manuscript. MF assisted with data review and interpretation and was a major contributor to writing the manuscript. JV played a major role in RECOVERY study participant recruitment and data collection and was a major contributor in writing the manuscript. EW and SF played major roles in study design, data review, and were major contributors in writing the manuscript. TR was a major contributor in RECOVERY study design, data analysis, interpretation and review, and writing the manuscript.

\section{Acknowledgements}

The authors would like to thank the RECOVERY study participants for their participation in this study.

\section{Authors' information (optional)}

Address Correspondence to: Rebecca Spigel, Division of Adolescent and Young Adult Medicine, Boston Children's Hospital, Boston, MA. Email Address:Rebecca.spigel@childrens.harvard.edu; 617-355-8456

\section{References}

1. Kaufman KR, Petkova E, Bhui KS, Schulze TG. A global needs assessment in times of a global crisis: world psychiatry response to the COVID-19 pandemic. BJPsych Open. 2020; doi.org/10.1192/bjo.2020.25.

2. American Psychiatric Association. Diagnostic and statistical manual of mental disorders. 5th Ed. 2013;doi.org/10.1176/appi.books.9780890425596. 
3. Termorshuizen JD, Watson HJ, Thornton LM, Borg S, Flatt RE, MacDermond CM, et al. Early impact of COVID-19 on individuals with self-reported eating disorders: A survey of $\sim 1,000$ individuals in the United States and the Netherlands. Int J Eat Disord. 2020; doi.org/10.1002/eat.23353.

4. Branley-Bell D, Talbot CV. Exploring the impact of the COVID-19 pandemic and UK lockdown on individuals with experience of eating disorders. J Eat Disord. 2020;doi.org/10.1186/s40337-02000319-y.

5. Schlegl S, Maier J, Meule A, Voderholzer U. Eating disorders in times of the COVID-19 pandemicResults from an online survey of patients with anorexia nervosa. Int J Eat Disord. 2020; doi.org/10.1002/eat.23374.

6. Rodgers RF, Lombardo C, Cerolini S, Franko DL, Omori M, Fuller-Tyszkiewicz M, et al. The impact of the COVID-19 pandemic on eating disorder risk and symptoms. Int J Eat Disord. 2020; doi.org/10.1002/eat.23318.

7. Bermudez O, Devlin M, Dooley-Hash S, Guarda A, Katzman DK, Madden S, et al. AED A Guide to Medical Care: Critical Points for Early Recognition and Medical Risk Management in the Care of Individuals with Eating Disorders, 3rd Ed. 2016. http://www.aedweb.org/Medical_Care_Standards.

8. Fernández-Aranda F, Casas M, Claes L, Bryan DC, Favaro A, Granero R, et al. COVID-19 and Implications for Eating Disorders. Eur Eat Disord Rev. 2020 May;doi: 10.1002/erv.2738.

9. Augenstein J. Trump Administration Makes Sweeping Regulatory Changes to Help U.S. Healthcare System Address COVID-19 Patient Surge (2020). https://www.healthaffairs.org/do/10.1377/hblog20200315.319008/full. Accessed 14 Dec 2020.

10. Davis C, Ng KC, Oh JY, Baeg A, Rajasegaran K, Chew CSE. Caring for Children and Adolescents With Eating Disorders in the Current Coronavirus 19 Pandemic: A Singapore Perspective. J Adolesc Health. 2020;doi: 10.1016/j.jadohealth.2020.03.037.

11. Barney A, Buckelew S, Mesheriakova V, Raymond-Flesch M. The COVID-19 Pandemic and Rapid Implementation of Adolescent and Young Adult Telemedicine: Challenges and Opportunities for Innovation. J Adolesc Health. 2020;doi: 10.1016/j.jadohealth.2020.05.006.

12. Waller G, Pugh M, Mulkens S, Moore E, Mountford VA, Carter J, et al. Cognitive-behavioral therapy in the time of coronavirus: Clinician tips for working with eating disorders via telehealth when face-toface meetings are not possible. Int J Eat Disord. 2020;doi: 10.1002/eat.23289.

13. Weissman RS, Bauer S, Thomas JJ. Access to evidence-based care for eating disorders during the COVID-19 crisis. Int J Eat Disord. 2020;doi: 10.1002/eat.23279.

14. Matheson BE, Bohon C, Lock J. Family-based treatment via videoconference: Clinical recommendations for treatment providers during COVID-19 and beyond. Int J Eat Disord. 2020; doi: 10.1002/eat.23326.

15. Golden NH, Katzman DK, Sawyer SM, Ornstein RM. Position paper of the society for adolescent health and medicine: Medical management of restrictive eating disorders in adolescents and young adults references. J Adolesc Health. 2015;doi: 10.1016/j.jadohealth.2014.10.259. 
16. Tremain H, McEnery C, Fletcher K, Murray G. The therapeutic alliance in digital mental health interventions for serious mental illnesses: Narrative review. JMIR Ment Health. 2020;doi: $10.2196 / 17204$.

17. Khosravi M. The challenges ahead for patients with feeding and eating disorders during the COVID19 pandemic. J Eat Disord. 2020;doi: 10.1186/s40337-020-00322-3.

18. Datta N, Derenne J, Sanders M, Lock JD. Telehealth transition in a comprehensive care unit for eating disorders: Challenges and long-term benefits. Int J Eat Disord. 2020;doi: 10.1002/eat.23348.

19. Wood SM, White K, Peebles R, Pickel J, Alausa M, Mehringer J, et al. Outcomes of a Rapid Adolescent Telehealth Scale-Up During the COVID-19 Pandemic. J Adolesc Health. 2020; doi:

10.1016/j.jadohealth.2020.05.025.

20. Graell M, Morón-Nozaleda MG, Camarneiro R, Villaseñor Á, Yáñez S, Muñoz R, et al. Children and adolescents with eating disorders during COVID-19 confınement: Difficulties and future challenges. Eur Eat Disord Rev. 2020;doi: 10.1002/erv.2763.

21. Gordon CM, Katzman DK. Lessons Learned in Caring for Adolescents With Eating Disorders: The Singapore Experience. J Adolec Health. 2020;doi: 10.1016/j.jadohealth.2020.03.041.

22. Backhaus A, Agha Z, Maglione ML, Repp A, Ross B, Zuest D, et al. Videoconferencing psychotherapy: A systematic review. Psychol Serv. 2012;doi: 10.1037/a0027924.

23. Myers K, Nelson EL, Rabinowitz T, Hilty D, Baker D, Barnwell SS, et al. American Telemedicine Association Practice Guidelines for Telemental Health with Children and Adolescents. Telemed J E Health. 2017;doi: 10.1089/tmj.2017.0177.

24. Sweeney GM, Donovan CL, March S, Forbes Y. Logging into therapy: Adolescent perceptions of online therapies for mental health problems. Internet Interv. 2016; doi: 10.1016/j.invent.2016.12.001.

25. Jenkins-Guarnieri MA, Pruitt LD, Luxton DD, Johnson K. Patient perceptions of telemental health: Systematic review of direct comparisons to in-person psychotherapeutic treatments. Telemed J E Health. 2015;doi: 10.1089/tmj.2014.0165.

26. Anderson KE, Byrne C, Goodyear A, Reichel R, Le Grange D. Telemedicine of family-based treatment for adolescent anorexia nervosa: A protocol of a treatment development study. J Eat Disord. 2015;doi: 10.1186/s40337-015-0063-1.

27. Anderson KE, Byrne CE, Crosby RD, Le Grange D. Utilizing Telehealth to deliver family-based treatment for adolescent anorexia nervosa. Int J Eat Disord. 2017;doi: 10.1002/eat.22759.

28. Holmes EA, O’Connor RC, Perry VH, Tracey I, Wessely S, Arseneault L, et al. Multidisciplinary research priorities for the COVID-19 pandemic: a call for action for mental health science. Lancet Psychiatry. 2020;doi: 10.1016/S2215-0366(20)30168-1.

29. Taylor CB, Fitzsimmons-Craft EE, Graham AK. Digital technology can revolutionize mental health services delivery: The COVID-19 crisis as a catalyst for change. Int J Eat Disord. 2020;doi: 10.1002/eat.23300.

30. Castellini G, Cassioli E, Rossi E, Innocenti M, Gironi V, Sanfilippo G, et al. The impact of COVID-19 Loading [MathJax]/jax/output/CommonHTML/jax.js dinal observation of pre versus post psychopathological 
features in a sample of patients with eating disorders and a group of healthy controls. Int $\mathrm{J}$ Eat Disord. 2020;doi: 10.1002/eat.23368.

31. Baenas I, Caravaca-Sanz E, Granero R, Sánchez I, Riesco N, Testa G, et al. COVID-19 and eating disorders during confinement: Analysis of factors associated with resilience and aggravation of symptoms. Eur Eat Disord Rev. 2020;doi: 10.1002/erv.2771.

32. McCombie C, Austin A, Dalton B, Lawrence V, Schmidt U. "Now It's Just Old Habits and Misery"Understanding the Impact of the Covid-19 Pandemic on People With Current or Life-Time Eating Disorders: A Qualitative Study. Front Psychiatry. 2020;doi.org/10.3389/fpsyt.2020.589225.

33. Clark Bryan D, Macdonald P, Ambwani S, Cardi V, Rowlands K, Willmott D, et al. Exploring the ways in which COVID-19 and lockdown has affected the lives of adult patients with anorexia nervosa and their carers. Eur Eat Disord Rev. 2020;doi: 10.1002/erv.2762.

34. Linville D, Brown T, Sturm K, McDougal T. Eating Disorders and Social Support: Perspectives of Recovered Individuals. Eat Disord. 2012;doi: 10.1080/10640266.2012.668480.

35. Brown M, Robinson L, Campione GC, Wuensch K, Hildebrandt T, Micali N. Intolerance of Uncertainty in Eating Disorders: A Systematic Review and Meta-Analysis. Eur Eat Disord Rev. 2017;doi: 10.1002/erv.2523.

36. Shah M, Sachdeva M, Johnston H. Eating disorders in the age of COVID-19. Psychiatry Res. 2020; doi: 10.1016/j.psychres.2020.113122.

37. Haddad C, Zakhour M, Bou Kheir M, Haddad R, Al Hachach M, Sacre H, et al. Association between eating behavior and quarantine/confinement stressors during the coronavirus disease 2019 outbreak. J Eat Disord. 2020;doi: 10.1186/s40337-020-00317-0. 